\title{
Psoas Compartment Block for Lower Limb Surgeries: A Comparative Study among The Four Lumbar Plexus Localizing Methods
}

\author{
Ahmed Mohamed Mahmoud Awadalla*, Adel Rizk Botros, Howida Kamal Abd Ellatif
}

Anesthesia and Intensive Care, Faculty of Medicine, Zagazig University, Zagazig, Egypt

*Corresponding author: Ahmed Mohamed Mahmoud Awadalla, Mobile: (+20) 01204420302, E-Mail: audy1980.aa@gmail.com

\begin{abstract}
Background: Psoas compartment block (PCB) is a frequently used techniques providing anesthesia for proximal lower limb surgeries. There are many methods of localization of peripheral nerves for regional anesthesia as loss of resistance (LOR), electric nerve stimulation (ENS), ultrasound-guided (US) and combined ultrasound-guided and electric nerve stimulation (US/ENS).

Objective: To compare between these four techniques to find out the method of better outcome and least complications. Patients and Methods: This study was a prospective randomized trial, conducted at Anesthesia and Intensive Care Department, Zagazig University Hospitals during the period from January 2018 to January, 2020. 140 patients undergoing unilateral elective proximal lower limb surgeries were included. Patients were categorized randomly, according to the used method for lumbar plexus localization, into four equal groups (each 35 patients). All groups were compared regarding efficacy and safety.

Results: The success rates of PCB in ENS, US and US/ENS groups were higher than that in LOR group with combined US/ENS group was the best group (97.14\%). The onset of sensory and motor blocks in ENS, US and US/ENS groups were shorter than that in LOR group. Times to ask for the $1^{\text {st }}$ postoperative analgesia in ENS, US and US/ENS groups were longer than those in LOR group with the consumed amount of morphine for pain relief during the $1^{\text {st }} 24$ hours postoperatively in ENS, US and US/ENS groups were lower than that in LOR group.
\end{abstract}

Conclusion: Usage of combined ultrasound and electric nerve stimulation was better regarding the outcome and complications.

Keywords: Electric nerve stimulation, Localizing methods, Psoas compartment block.

\section{INTRODUCTION}

Nowadays, the interest of peripheral nerve blocks (PNBs) to provide anesthesia/or analgesia for lower limb surgeries have increased as central neuraxial blocks (CNBs) are associated with many complications as transient neurologic symptoms with spinal block and epidural hematoma with epidural block ${ }^{(1)}$. Psoas compartment block (PCB) is one of the frequently used techniques for providing anesthesia/or analgesia in cases of proximal lower limb surgical procedures. It blocks all lumbar nerves' roots and potentially some sacral nerves' roots via posterior approach ${ }^{(2)}$.

PCB has some advantages as providing efficient intraoperative anesthesia, excellent postoperative analgesia and stable hemodynamics especially in elder patients ${ }^{(3)}$. If anesthesia or analgesia of the lower leg or posterior thigh is required for the lower limb surgical procedure, a sciatic nerve block must be added to psoas compartment block ${ }^{(4)}$.

Block of the various lumbar plexus nerve roots after injection of the selected local anesthetic (LA) solution within the psoas compartment (PC) is due to the cephalad and caudate spread of the injected LA solution (5). In $25 \%$ of cases, block of the first sacral nerve root also occurs and in $70 \%$ of cases an ilioinguinal/iliohypogastric block is found ${ }^{\left({ }^{6}\right)}$.

The commonly used methods of localization of peripheral nerves for regional anesthesia involve anatomical localization, eliciting of paresthesia, pops or clicks sensation and loss of resistance, electric nerve stimulation with either insulated or non-insulated needle, ultrasound-guided and combined ultrasound-guided and electric nerve stimulation ${ }^{(7)}$.

So, the current study was done to compare between loss of resistance (LOR), electrical nerve stimulation (ENS), ultrasound (US), and combined ultrasound and electrical nerve stimulation (US/ENS) as aiding methods for localizing lumbar plexus nerves during performance of a single-shot psoas compartment block to provide anesthesia for ipsilateral elective proximal lower limb surgical procedures in order to find out the method of better outcome and least complications.

\section{PATIENTS AND METHODS}

This study was a prospective randomized clinical trial. It was conducted at Anesthesia and Surgical Intensive Care Department, Zagazig University Hospitals during the period from January 2018 to January, 2020.

\section{Ethical approval:}

This study was approved by Zagazig University's Institutional Review Board. A written informed consent was obtained from all patients participating in the study. Also, this study was carried out according to the Code of Ethics of the World Medical Association (Declaration of Helsinki) for studies involving humans.

Inclusion criteria were cooperative both sexes patients, their ages ranged from 21 to 60 years, their body mass 
index (BMI) ranged from 18.5 to $29.9 \mathrm{~kg} / \mathrm{m}^{2}$, their physical status, according to ASA classification, ranged from class I to class II, and undergoing unilateral elective proximal lower limb surgical procedures. Exclusion criteria were refusal of PCB, history of allergy to LA, bleeding tendency, coagulopathy, anticoagulant therapy, pre-existing psychological, neurologic and spinal cord diseases, previous back surgery, infection or mass at the site of the block, chronic analgesic therapy and severe cardiac or renal diseases.

\section{Randomization and categorization:}

Patients were categorized randomly, according to the used method for lumbar plexus localization during performance of PCB, using closed envelope technique, into four equal groups (each 35 patients). These groups were the following: Loss of resistance (LOR) group (using Tuohy needle insertion), Electrical nerve stimulation (ENS) group, Ultrasound (US) group and combined ultrasound and electrical needle stimulation (US/ENS) group.

Preoperatively, patients' management included preoperative clinical assessment, preparation and premedication.

Preoperative clinical assessment involved the following: (i) History taking with special attention to any medical problems especially muscle or spine disease, renal or liver impairment, previous back surgeries and the type of the used anesthesia, any psychological or neurological disorders, allergy to the used LA, bleeding disorders, medications (as anticoagulants, antiepileptic, etc.), (ii) Thorough physical examination and examination of patient's back and the site of needle insertion to roll out the presence of any contraindication as infection, mass, deformity of vertebral column ...etc., (iii) Laboratory investigations included CBC, coagulation profile, liver function tests, kidney function tests and random blood sugar level, and ECG (for age above 40 years).

Preoperative patients' preparation included control of any medical problems, explanation of PCB technique to patient, prescribing pre-medication and giving instruction for the fasting period if an elective surgical procedure was planned.

Pre-medication involved giving $1 \mathrm{mg}$ atropine sulfate IM 1 hour before surgery and midazolam $(10 \mu \mathrm{cg} / \mathrm{kg})$ and fentanyl $(1 \mu \mathrm{g} / \mathrm{kg})$ iv before starting psoas compartment block.

Measure for patients' safety during $P C B$ involved performing all $\mathrm{PCBs}$ in operating room (OR) and also the resuscitation equipment $\left(\mathrm{O}_{2}\right.$, suction, ventilation bag with valve and mask, intubation's set for urgent intubation and defibrillator), emergency drugs (atropine, epinephrine, ephedrine, antiarrhythmic drugs) and IV fluids were available and near hand.

On arrival of the patient to $O R$, blood pressure cuff, pulse oximeter probe and ECG leads were applied to the patient, a 16-18 gauge iv cannula was inserted and secured then lactated Ringer solution $(500 \mathrm{ml})$ was administered. Also, supplemental oxygen (4-6 L/min) through face mask was administered to patients during operation.

In this study, technical procedures for performing Single-shot PCB, included 7 steps. These steps were the following: (1) Preparation of standard regional anesthesia tray beside each of Touhy needle for LOR group, electrical nerve stimulator for ENS group and ultrasound machine for US group or both for US/ENS group, (2) Putting patient in lateral decubitus position with the operative side uppermost and the hips and knees are flexed to $90^{\circ}$, (3) Application of all aseptic measures, (4) Determination of the point of needle insertion according to Capdevila et $\boldsymbol{a l}$. approach ${ }^{(2)}$, A line was drawn from the center of the L4 spinous process laterally toward the iliac crest of nondependent side, to intersect with a line that passes through the posterior superior iliac spine (PSIS) of the nondependent side parallel to the vertebral column on the side to be blocked. The point of needle insertion was at the junction of the lateral one third and medial two thirds of the line joining the center of the L4 spinous process to the line passing through PSIS and parallel to spinal line (5) LA skin infiltration at the site of needle insertion, (6) Localization of lumbar plexus within PC by one of the four methods chosen for the study (LOR, ENS, US and US/ENS) and (7) Single shot injection of the $40 \mathrm{ml}$ of LA mixture $(20 \mathrm{ml}$ of $0.5 \%$ bupivacaine plus $20 \mathrm{ml}$ of $2 \%$ lidocaine and both with 1:200,000 epinephrine) after repeated negative aspiration for blood and cerebrospinal fluid (CSF).

\section{Statistical analysis}

Data collected were coded, entered and analyzed using Microsoft Excel software. Data were then imported into Statistical Package for the Social Sciences (SPSS version 20.0) software for analysis. Qualitative data were represented as number and percentage, while, quantitative data were represented by mean \pm standard deviation (SD) and range. The following tests were used: difference and association of qualitative variable by Chi square test $\left(\mathrm{X}^{2}\right)$, while, differences between quantitative independent multiple groups by ANOVA or Kruskal Wallis test, and by repeated measurement ANOVA for related means. P-value at $<0.05$ was considered significant difference and $<0.001$ was considered highly significant difference, and when indicates significance the post hoc Tukey's test was used to compare specific data between groups.

\section{RESULTS}

Statistically, patients' characteristics (age, weight, height, BMI, sex ratio, and ASA Ps classes, types and duration of surgery were comparable in the four studied groups with no significant difference (Table 1). 
Table (1): Patients' demographic data, distribution of the various types of operations and duration of surgery in the four studied groups

\begin{tabular}{|c|c|c|c|c|c|c|}
\hline \multirow[t]{2}{*}{ Variable } & \multirow{2}{*}{$\begin{array}{c}\text { LOR } \\
\text { Group }(n=35)\end{array}$} & \multirow{2}{*}{$\begin{array}{c}\text { ENS } \\
\text { Group }(n=35)\end{array}$} & \multirow{2}{*}{$\begin{array}{c}\mathrm{US} \\
\operatorname{group}(\mathrm{n}=35)\end{array}$} & \multirow{2}{*}{$\begin{array}{c}\text { US/ENS } \\
\operatorname{group}(n=35)\end{array}$} & \multicolumn{2}{|c|}{ Test } \\
\hline & & & & & $f / x^{2}$ & value \\
\hline Age (years) & $44.06 \pm 8.57$ & $43.64 \pm 9.15$ & $44.08 \pm 10.9$ & $\begin{array}{c}43.64 \pm \\
8.44\end{array}$ & 0.025 & 0.99 \\
\hline Weight (kg) & $80.43 \pm 6.25$ & $81.2 \pm 5.12$ & $79.9 .21 \pm 6.85$ & $80.45 \pm 6.08$ & 0.290 & 0.83 \\
\hline Height (cm) & $170.4 \pm 5.67$ & $170.2 \pm 4.6$ & $169.7 \pm 5.3$ & $169.8 \pm 6.4$ & 0.125 & 0.94 \\
\hline BMI $\left(\mathrm{kg} / \mathrm{m}^{2}\right)$ & $24.3 \pm 3.5$ & $24.7 \pm 2.5$ & $23.5 \pm 3.5$ & $24.15 \pm 2.75$ & 0.910 & 0.438 \\
\hline Sex (Males/Females) & $21 / 14$ & $20 / 15$ & $16 / 16$ & $18 / 17$ & 1.69 & 0.638 \\
\hline ASA Ps classes (I/II) & $15 / 20$ & $12 / 23$ & $16 / 19$ & $14 / 21$ & 1.036 & 0.793 \\
\hline $\begin{array}{l}\text { Distribution of the } \\
\text { various types of } \\
\text { operations (N): } \\
\text { - Total knee } \\
\text { arthroplasty. }\end{array}$ & 4 & 5 & 5 & 5 & 0.183 & 0.98 \\
\hline $\begin{array}{l}\text { - Bipolar hip } \\
\text { hemiarthroplasty }\end{array}$ & 5 & 4 & 6 & 6 & 0.616 & 0.893 \\
\hline $\begin{array}{l}\text {-Internal fixation of } \\
\text { Fracture acetabulum }\end{array}$ & 6 & 5 & 4 & 5 & 0.168 & 0.983 \\
\hline $\begin{array}{l}\text { - Closed reduction and } \\
\text { internal fixation of } \\
\text { fracture femur }\end{array}$ & 8 & 9 & 8 & 8 & 0.119 & 0.989 \\
\hline - Knee arthroscopy & 12 & 12 & 12 & 11 & 0.096 & 0.992 \\
\hline $\begin{array}{l}\text { Duration of surgery } \\
\text { (min) }\end{array}$ & $122.62 \pm 14.4$ & $120.6 \pm 14.3$ & $121.5 \pm 14.7$ & $123.2 \pm 13.9$ & 0.257 & 0.856 \\
\hline
\end{tabular}

Data are expressed as mean \pm SD or numbers.

$\mathrm{n}=$ Group number.

Regarding psoas compartment block's performance times, the mean performance times in US/ENS and US groups were comparable but significantly longer than the mean times in ENS and LOR groups. The mean of discomfort scores in ENS and US/ENS groups were comparable but significantly higher than those in LOR and US groups. Statistically, the means of the numbers of the blocked nerves from both lumbar and sacral plexuses at $30 \mathrm{~min}$ after injection of local anesthetic mixture in ENS, US and US/ENS groups were significantly higher than that in LOR group. The mean of the numbers of the blocked nerves in US and US/ENS groups were comparable and significantly higher than that in ENS group. Regarding the success rate of psoas compartment block, the success rates of PCB in ENS, US and US/ENS groups were significantly higher than that in LOR group. Also, success rates in US and US/ENS groups were significantly higher than that in ENS group and success rate in US/ENS group was significantly higher than that in US group (Table 2). 
Table (2): Psoas compartment block's performance times, the levels of patients' discomfort, the maximal block extension levels and Success rates during lumber plexus localization in the four studied groups

\begin{tabular}{|c|c|c|c|c|c|c|c|}
\hline & \multirow{2}{*}{$\begin{array}{c}\begin{array}{c}\text { LOR } \\
\text { group } \\
(\mathrm{n}=35)\end{array} \\
\text { Mean } \pm \text { SD }\end{array}$} & \multirow{2}{*}{\begin{tabular}{|c|}
$\begin{array}{c}\text { ENS } \\
\text { Group } \\
(\mathbf{n}=35)\end{array}$ \\
Mean \pm SD \\
\end{tabular}} & \multirow{2}{*}{$\begin{array}{c}\begin{array}{c}\text { US } \\
\text { Group } \\
(n=35)\end{array} \\
\text { Mean } \pm \text { SD }\end{array}$} & \multirow{2}{*}{\begin{tabular}{|c|}
$\begin{array}{c}\text { US/ENS } \\
\text { Group } \\
(\mathbf{n}=35)\end{array}$ \\
Mean \pm SD
\end{tabular}} & \multicolumn{2}{|c|}{$\begin{array}{c}\text { One-way } \\
\text { ANOVA test }\end{array}$} & \multirow{2}{*}{$\begin{array}{l}\text { The post } \\
\text { hoc } \\
\text { Tukey's tes }\end{array}$} \\
\hline & & & & & $f$ & $P$ value & \\
\hline $\begin{array}{l}\text { Block } \\
\text { performance } \\
\text { time (min). }\end{array}$ & $8.22 \pm 0.91$ & $8.82 \pm 1.24$ & $10.05 \pm 1.7$ & $10.94 \pm 1.5$ & 27.115 & $<0.001$ & $\begin{array}{l}\mathrm{P} 1=0.26 \\
\mathrm{P} 2<0.001 \\
\mathrm{P} 3<0.001 \\
\mathrm{P} 4<0.001 \\
\mathrm{P} 5<0.001 \\
\mathrm{P} 6=0.25\end{array}$ \\
\hline $\begin{array}{l}\text { Discomfort } \\
\text { scores }\end{array}$ & $\begin{array}{c}0-2 \\
(0.51 \pm 0.1 \\
5)\end{array}$ & $\begin{array}{c}0-2 \\
(1.28 \pm 0.43)\end{array}$ & $\begin{array}{c}0-2 \\
(0.57 \pm 0.17 \\
)\end{array}$ & $\begin{array}{c}0-2 \\
(1.2 \pm 0.4)\end{array}$ & 24.5 & $<0.001$ & $\begin{array}{l}\mathrm{P} 1<0.001 \\
\mathrm{P} 2=0.634 \\
\mathrm{P} 3<0.001 \\
\mathrm{P} 4=0.001 \\
\mathrm{P} 5=0.47 \\
\mathrm{P} 6<0.001\end{array}$ \\
\hline $\begin{array}{l}\text { Number of } \\
\text { blocked nerves } \\
\text { out of the main } \\
9 \text { nerves of both } \\
\text { lumber and } \\
\text { sacral plexuses. }\end{array}$ & $\begin{array}{c}2-7 \\
(5.5 \pm 1.25)\end{array}$ & $\begin{array}{c}4-8 \\
(7.21 \pm 0.54)\end{array}$ & $\begin{array}{c}4-9 \\
(8.10 \pm 0.56 \\
)\end{array}$ & $\begin{array}{c}2-9 \\
(8.25 \pm 0.5)\end{array}$ & 95.9 & $<0.001$ & $\begin{array}{l}\mathrm{P} 1<0.001 \\
\mathrm{P} 2<0.001 \\
\mathrm{P} 3<0.001 \\
\mathrm{P} 4<0.001 \\
\mathrm{P} 5<0.001 \\
\mathrm{P} 6=0.241\end{array}$ \\
\hline Success rate & $\begin{array}{c}26 \\
(74.28 \%)\end{array}$ & $\begin{array}{c}30 \\
(85.71 \%)\end{array}$ & $\begin{array}{c}32 \\
(91.42 \%)\end{array}$ & $\begin{array}{c}34 \\
(97.14 \%)\end{array}$ & 23.52 & 0.03 & $\begin{array}{l}\mathrm{P} 1=0.03 \\
\mathrm{P} 2=0.02 \\
\mathrm{P} 3=0.013 \\
\mathrm{P} 4=0.04 \\
\mathrm{P} 5=0.03 \\
\mathrm{P} 6=0.04\end{array}$ \\
\hline
\end{tabular}

$\mathrm{n}=$ Group number.

Data are expressed as range and mean \pm SD or as number and percentage (No (\%)).

$\mathrm{P} 1=$ LOR group vs. ENS group. P2=LOR group vs. US group.

$\mathrm{P} 3=\mathrm{LOR}$ group vs. US/ENS group.

$\mathrm{P} 4=\mathrm{ENS}$ group vs. US group.

P5= ENS group vs. US/ENS group. $\quad$ P6= US group vs. US/ENS group.

It was found that the onset of sensory and motor blocks in ENS, US and US/ENS groups were significantly shorter than that in LOR group. Also, the onset of sensory and motor blocks in US and US/ENS groups were comparable and shorter than that in ENS group (Table 3). 
Table (3): Onset of sensory and motor blocks in the four studied groups

\begin{tabular}{|c|c|c|c|c|c|c|c|}
\hline & \multirow{2}{*}{$\begin{array}{c}\begin{array}{c}\text { LOR } \\
\text { Group } \\
(\mathbf{n}=\mathbf{2 6})\end{array} \\
\text { Mean } \pm \text { SD }\end{array}$} & \multirow{2}{*}{$\begin{array}{c}\begin{array}{c}\text { ENS } \\
\text { Group } \\
(\mathbf{n}=\mathbf{3 0})\end{array} \\
\text { Mean } \pm \text { SD } \\
\end{array}$} & \multirow{2}{*}{$\begin{array}{c}\begin{array}{c}\text { US } \\
\text { Group } \\
(n=32)\end{array} \\
\text { Mean } \pm \text { SD } \\
\end{array}$} & \multirow{2}{*}{$\begin{array}{c}\begin{array}{c}\text { US/ENS } \\
\text { Group } \\
(n=34)\end{array} \\
\text { Mean } \pm \text { SD }\end{array}$} & \multicolumn{2}{|c|}{$\begin{array}{c}\text { One-way } \\
\text { ANOVA test }\end{array}$} & \multirow[t]{2}{*}{$\begin{array}{l}\text { The post hoc } \\
\text { Tukey's test }\end{array}$} \\
\hline & & & & & $f$ & $P$ value & \\
\hline $\begin{array}{l}\text { Onset of } \\
\text { sensory block } \\
\text { (min) }\end{array}$ & $15.25 \pm 3.5$ & $12.75 \pm 3.65$ & $10.50 \pm 2.5$ & $10.25 \pm 2.40$ & 19.84 & $<0.001$ & $\begin{array}{l}\mathrm{P} 1=0.005 \\
\mathrm{P} 2<0.001 \\
\mathrm{P} 3<0.001 \\
\mathrm{P} 4=0.004 \\
\mathrm{P} 5<0.001 \\
\mathrm{P} 6=0.671\end{array}$ \\
\hline $\begin{array}{l}\text { Onset of motor } \\
\text { block } \\
\text { (min) }\end{array}$ & $23.73 \pm 3.45$ & $19.1 \pm 3.76$ & $16.41 \pm 3.71$ & $15.21 \pm 3.35$ & 38.93 & $<0.001$ & $\begin{array}{l}\mathrm{P} 1<0.001 \\
\mathrm{P} 2<0.001 \\
\mathrm{P} 3<0.001 \\
\mathrm{P} 4=0.004 \\
\mathrm{P} 5<0.001 \\
\mathrm{P} 6=0.155\end{array}$ \\
\hline $\begin{array}{l}\text { OR group vs. } \\
\text { LOR group vs. } \\
\text { ENS group vs. }\end{array}$ & $\begin{array}{l}\text { group. } \\
\text { EN group. } \\
\text { ENS group. }\end{array}$ & & $\begin{array}{l}\mathrm{n}=\text { Group nun } \\
\mathrm{P} 2=\text { LOR gro } \\
\mathrm{P} 4=\text { ENS gro } \\
\mathrm{P} 6=\text { US grou }\end{array}$ & $\begin{array}{l}\text { vs. US group. } \\
\text { s. US group. } \\
\text { s. US/ENS grc }\end{array}$ & & & \\
\hline
\end{tabular}

Statistically, the means of the intraoperative pain severity scores were comparable and below 1 in the patients of the four studied groups. For this reason, supplementary iv fentanyl was not needed for intraoperative pain relief. Times to ask for the $1^{\text {st }}$ postoperative analgesia in ENS, US and US/ENS groups were significantly longer than those in LOR group. Also, times to ask for the $1^{\text {st }}$ postoperative analgesia in US and US/ENS groups were comparable and significantly longer than that in ENS group. Statistically, the consumed amount of morphine for pain relief during the $1^{\text {st }} 24$ hours postoperatively in ENS, US and US/ENS groups were significantly lower than that in LOR group. Also, the consumed amount of morphine in US and US/ENS groups were comparable and significantly lower than that in ENS group (Table 4).

Table (4): Time to ask for the first postoperative analgesia and the total morphine consumption in the first postoperative day in the four studied groups

\begin{tabular}{|c|c|c|c|c|c|c|c|}
\hline & \multirow{2}{*}{$\begin{array}{c}\begin{array}{c}\text { LOR } \\
\text { Group } \\
(\mathbf{n}=35)\end{array} \\
\text { Mean } \pm \text { SD }\end{array}$} & \multirow{2}{*}{$\begin{array}{c}\begin{array}{c}\text { ENS } \\
\text { Group } \\
(n=35)\end{array} \\
\text { Mean } \pm \text { SD }\end{array}$} & \multirow{2}{*}{$\begin{array}{c}\begin{array}{c}\text { US } \\
\text { Group } \\
(\mathbf{n}=35)\end{array} \\
\text { Mean } \pm \text { SD }\end{array}$} & \multirow{2}{*}{$\begin{array}{c}\begin{array}{c}\text { US/ENS } \\
\text { Group } \\
(\mathrm{n}=35)\end{array} \\
\text { Mean } \pm \text { SD }\end{array}$} & \multicolumn{2}{|c|}{$\begin{array}{c}\text { One-way } \\
\text { ANOVA test }\end{array}$} & \multirow{2}{*}{$\begin{array}{c}\text { The post } \\
\text { hoc } \\
\text { Tukey's } \\
\text { test }\end{array}$} \\
\hline & & & & & $f$ & $P$ value & \\
\hline $\begin{array}{l}\text { The time to ask } \\
\text { for the first post- } \\
\text { operative } \\
\text { analgesia (hrs.) }\end{array}$ & $2.05 \pm 0.64$ & $2.45 \pm 0.71$ & $3.35 \pm 0.91$ & $3.45 \pm 0.83$ & 27.002 & $<0.001$ & $\begin{array}{l}\mathrm{P} 1=0.016 \\
\mathrm{P} 2<0.001 \\
\mathrm{P} 3<0.001 \\
\mathrm{P} 4<0.001 \\
\mathrm{P} 5<0.001 \\
\mathrm{P} 6=0.633\end{array}$ \\
\hline $\begin{array}{l}\text { Total morphine } \\
\text { consumption in } \\
\text { 1st postoperative } \\
\text { day (mg/patient) }\end{array}$ & $12.66 \pm 2.69$ & $10.16 \pm 2.06$ & $8.77 \pm 2.4$ & $8.56 \pm 1.45$ & 25.77 & $<0.001$ & $\begin{array}{l}\mathrm{P} 1<0.001 \\
\mathrm{P} 2<0.001 \\
\mathrm{P} 3<0.001 \\
\mathrm{P} 4=0.003 \\
\mathrm{P} 5<0.001 \\
\mathrm{P} 6=0.695\end{array}$ \\
\hline
\end{tabular}

Data are expressed as mean \pm SD

P1 =LOR group vs. ENS group.

P3 =LOR group vs. US/EN group.

$\mathrm{P} 5=$ ENS group vs. US/ENS group. $\mathrm{n}=$ Group number.

P2 =LOR group vs. US group.

P4 =ENS group vs. US group.

P6 = US group vs. US/ENS group.

Regarding hemodynamic and respiratory changes, the mean values of mean arterial pressure, heart rate, respiratory rate and peripheral oxygen saturation at various times of measurements in the four studied groups showed no significant difference with their corresponding baseline values (Tables 5 and 6). 
Table (5): Hemodynamic changes in the four studied groups

\begin{tabular}{|c|c|c|c|c|c|}
\hline & \multirow{2}{*}{\multicolumn{2}{|c|}{$\begin{array}{c}\begin{array}{c}\text { LOR group } \\
(\mathrm{n}=26)\end{array} \\
\text { Mean } \pm \text { SD }\end{array}$}} & $\begin{array}{c}\text { ENS group } \\
(n=30)\end{array}$ & $\begin{array}{c}\text { US group } \\
(\mathrm{n}=32)\end{array}$ & $\begin{array}{c}\text { US/ENS group } \\
(n=34)\end{array}$ \\
\hline & & & Mean \pm SD & Mean \pm SD & Mean \pm SD \\
\hline $\begin{array}{l}\text { Mean arterial pressure } \\
\text { (mmHg): } \\
\text { Base line value. }\end{array}$ & \multicolumn{2}{|c|}{$82.1 \pm 5.2$} & $84.9 \pm 4.4$ & $81 \pm 2.5$ & $79.5 \pm 3.9$ \\
\hline $\begin{array}{l}\text { After local anesthetic } \\
\text { injection: } \\
15 \text { min. } \\
30 \mathrm{~min} . \\
45 \mathrm{~min} . \\
60 \mathrm{~min} .\end{array}$ & \multicolumn{2}{|c|}{$\begin{array}{c}81.7 \pm 4.3 \\
81.4 \pm 5 \\
81.2 \pm 5.3 \\
82.4 \pm 5.4\end{array}$} & $\begin{array}{l}81.6 \pm 4.3 \\
82.4 \pm 4.2 \\
82.6 \pm 3.9 \\
83.6 \pm 3.9\end{array}$ & $\begin{array}{c}79.7 \pm 4.1 \\
80.6 \pm 4.1 \\
82.6 \pm 3.9 \\
82.6 \pm 3.9\end{array}$ & $\begin{array}{l}77.7 \pm 3.6 \\
78.2 \pm 3.6 \\
79.5 \pm 3.4 \\
80.5 \pm 3.4\end{array}$ \\
\hline \multirow{2}{*}{$\begin{array}{l}\text { Repeated measurement } \\
\text { ANOVA test }\end{array}$} & $f$ & 0.277 & 1.22 & 1.66 & 1.27 \\
\hline & $P$ value & 0.87 & 0.303 & 0.160 & 0.281 \\
\hline $\begin{array}{l}\text { Heart rate } \\
\text { (beat/min): } \\
\text { Base line value. }\end{array}$ & \multicolumn{2}{|c|}{$81.7 \pm 4.8$} & $81.4 \pm 4.9$ & $80.4 \pm 4.9$ & $80.2 \pm 4.2$ \\
\hline $\begin{array}{l}\text { After local anesthetic } \\
\text { injection: } \\
15 \mathrm{~min} . \\
30 \mathrm{~min} \\
45 \mathrm{~min} . \\
60 \mathrm{~min} .\end{array}$ & \multicolumn{2}{|c|}{$\begin{array}{c}79.4 \pm 4.9 \\
78.3 \pm 5.2 \\
81.8 \pm 5.8 \\
81.4 \pm 4.2\end{array}$} & $\begin{array}{l}80.2 \pm 3.9 \\
80.6 \pm 1.7 \\
81.2 \pm 3.6 \\
81.5 \pm 2.3\end{array}$ & $\begin{array}{l}79.7 \pm 4.8 \\
79.7 \pm 4.8 \\
81.2 \pm 4.8 \\
80.7 \pm 4.8\end{array}$ & $\begin{array}{l}79.8 \pm 4.3 \\
80.8 \pm 4.3 \\
80.8 \pm 4.3 \\
81.8 \pm 4.3\end{array}$ \\
\hline \multirow{2}{*}{$\begin{array}{l}\text { Repeated measurement } \\
\text { ANOVA test }\end{array}$} & $f$ & 1.85 & 1.98 & 1.22 & 1.876 \\
\hline & $P$ value & 0.326 & 0.345 & 0.309 & 0.45 \\
\hline
\end{tabular}

$\mathrm{n}=$ Group number.

Data are expressed as mean \pm Standard Deviation (SD).

Table (6): Respiratory changes in the four studied groups

\begin{tabular}{|c|c|c|c|c|c|}
\hline & \multicolumn{2}{|c|}{$\begin{array}{c}\text { LOR group } \\
(\mathrm{n}=26)\end{array}$} & $\begin{array}{c}\text { ENS } \\
\text { group } \\
(\mathrm{n}=\mathbf{3 0}) \\
\end{array}$ & $\begin{array}{c}\text { US group } \\
(\mathrm{n}=32)\end{array}$ & $\begin{array}{c}\text { US/ENS group } \\
(n=34)\end{array}$ \\
\hline & \multicolumn{2}{|c|}{ Mean \pm SD } & Mean \pm SD & Mean \pm SD & Mean \pm SD \\
\hline $\begin{array}{l}\text { Respiratory rate (cycle/min): } \\
\text { Base line value. }\end{array}$ & \multicolumn{2}{|c|}{$12.7 \pm 1$} & $13.2 \pm 0.9$ & $13.2 \pm 0.8$ & $13.4 \pm 0.9$ \\
\hline $\begin{array}{l}\text { After local anesthetic injection: } \\
15 \mathrm{~min} . \\
30 \mathrm{~min} . \\
45 \mathrm{~min} . \\
60 \mathrm{~min} .\end{array}$ & \multicolumn{2}{|c|}{$\begin{array}{l}12.9 \pm 1.8 \\
13.3 \pm 1.2 \\
13.2 \pm 1.0 \\
12.7 \pm 1.0\end{array}$} & $\begin{array}{l}13.1 \pm 1.2 \\
12.8 \pm 1.3 \\
14.1 \pm 1.4 \\
13.2 \pm 1.4\end{array}$ & $\begin{array}{l}12.8 \pm 0.8 \\
13.4 \pm 1.1 \\
13.2 \pm 1.1 \\
12.9 \pm 1.1\end{array}$ & $\begin{array}{l}13.8 \pm 0.8 \\
13.6 \pm 0.9 \\
13.6 \pm 0.9 \\
13.5 \pm 1.0\end{array}$ \\
\hline \multirow{2}{*}{ Repeated measurement ANOVA test } & $f$ & 1.45 & 0.210 & 0.230 & 0.21 \\
\hline & $P$ value & 0.45 & 0.68 & 0.65 & 0.71 \\
\hline $\begin{array}{l}\mathrm{SpO}_{2}\left(\mathrm{O}_{2} \text { Saturation } \%\right): \\
\text { Base line value. }\end{array}$ & \multicolumn{2}{|c|}{$96.8 \pm 0.8$} & $96.9 \pm 1.0$ & $96.8 \pm 0.9$ & $96.7 \pm 1.0$ \\
\hline $\begin{array}{l}\text { After local anesthetic injection: } \\
15 \mathrm{~min} . \\
30 \mathrm{~min} . \\
45 \mathrm{~min} \\
60 \mathrm{~min} .\end{array}$ & \multicolumn{2}{|c|}{$\begin{array}{l}96.2 \pm 10 \\
96.7 \pm 1.2 \\
96.6 \pm 0.9 \\
96.8 \pm 0.9\end{array}$} & $\begin{array}{c}96.7 \pm 1.1 \\
95.6 \pm 1.3 \\
96.6 \pm 1.0 \\
96.6 \pm 0.9\end{array}$ & $\begin{array}{l}95.7 \pm 1.3 \\
97 \pm 1.10 \\
96.6 \pm 0.8 \\
96.6 \pm 1.1\end{array}$ & $\begin{array}{l}96.7 \pm 1.3 \\
95.9 \pm 1.3 \\
97.1 \pm 1.1 \\
97.3 \pm 1.1\end{array}$ \\
\hline \multirow[t]{2}{*}{ Repeated measurement ANOVA test } & $f$ & 0.199 & 0.12 & 0.21 & 0.31 \\
\hline & $P$ value & 0.88 & 0.98 & 0.76 & 0.82 \\
\hline
\end{tabular}

$\mathrm{n}=$ Group number.

Data are expressed mean \pm SD

Regarding the associated complications, the incidences of ipsilateral thoracic epidural blocks and contralateral femoral nerve and sacral plexus blocks in the four groups were comparable, but the incidences of vascular punctures in LOR and ENS were significantly higher than those in US and US/ENS groups (Table 7). 
Table (7): The incidences of the various associated complications in the four studied groups

\begin{tabular}{|c|c|c|c|c|c|}
\hline & $\begin{array}{c}\text { LOR } \\
\text { group } \\
(\mathrm{n}=26)\end{array}$ & $\begin{array}{c}\begin{array}{c}\text { ENS } \\
\text { group } \\
(\mathbf{n = 3 0})\end{array} \\
\operatorname{No}(\%)\end{array}$ & $\begin{array}{c}\text { US } \\
\text { group } \\
(\mathbf{n}=32)\end{array}$ & $\begin{array}{l}\text { US/ENS } \\
\text { group } \\
(\mathrm{n}=34) \\
\end{array}$ & $\begin{array}{c}\text { Chi-square } \\
\text { Test }\end{array}$ \\
\hline $\begin{array}{l}\text { Ipsilateral thoracic epidural } \\
\text { block. }\end{array}$ & $1(3.8 \%)$ & $1(3.3 \%)$ & $2(6.25 \%)$ & $2(5.88 \%)$ & $\begin{array}{l}\mathrm{P} 1=0.940 \\
\mathrm{P} 2=0.820 \\
\mathrm{P} 3=0.840 \\
\mathrm{P} 4=0.850 \\
\mathrm{P} 5=0.857 \\
\mathrm{P} 6=0.970\end{array}$ \\
\hline $\begin{array}{l}\text { Contralateral femoral nerve } \\
\text { block. }\end{array}$ & $1(3.8 \%)$ & $1(3.3 \%)$ & $2(6.25 \%)$ & $2(5.88 \%)$ & $\begin{array}{l}\mathrm{P} 1=0.940 \\
\mathrm{P} 2=0.820 \\
\mathrm{P} 3=0.840 \\
\mathrm{P} 4=0.850 \\
\mathrm{P} 5=0.857 \\
\mathrm{P} 6=0.970\end{array}$ \\
\hline $\begin{array}{l}\text { Contralateral sacral plexus } \\
\text { block. }\end{array}$ & $1(3.8 \%)$ & $1(3.3 \%)$ & $2(6.25 \%)$ & $2(5.88 \%)$ & $\begin{array}{l}\mathrm{P} 1=0.940 \\
\mathrm{P} 2=0.820 \\
\mathrm{P} 3=0.840 \\
\mathrm{P} 4=0.850 \\
\mathrm{P} 5=0.857 \\
\mathrm{P} 6=0.970\end{array}$ \\
\hline Vascular puncture. & $3(11.5 \%)$ & $3(9.9 \%)$ & $1(3.12 \%)$ & $1(2.9 \%)$ & $\begin{array}{l}\mathrm{P} 1=0.800 \\
\mathrm{P} 2=0.040 \\
\mathrm{P} 3=0.030 \\
\mathrm{P} 4=0.043 \\
\mathrm{P} 5=0.033 \\
\mathrm{P} 6=0.965\end{array}$ \\
\hline $\begin{array}{l}=\text { Group number. } \\
1=\text { LOR group vs. ENS group. } \\
3=\text { LOR group vs. US/ENS group. } \\
5=\text { ENS group vs. US/ENS group. }\end{array}$ & & $\begin{array}{l}\text { xpressed a } \\
\text { OR group } \\
\text { NS group v } \\
\text { S group v }\end{array}$ & $\begin{array}{l}\text { mber or perc } \\
\text { IS group. } \\
\text { S group. } \\
\text { S/ENS group }\end{array}$ & ge (No (\%) & \\
\hline
\end{tabular}

\section{DISCUSSION}

Psoas compartment block (PCB) is a peripheral regional anesthetic technique that blocks all lumbar nerves and potentially some sacral nerves to produce anesthesia or analgesia of proximal lower limbs ${ }^{(4)}$. In the present study, it was found that, the mean psoas compartment block performance times in US/ENS and US groups were comparable and significantly longer than the mean times in ENS and LOR groups. Also, the mean psoas compartment block performance times in LOR and ENS groups were comparable. These present study findings were in agreement with the reported findings of some workers. Danelli et al. ${ }^{(8)}$ reported that, psoas compartment block performance times with the aid of each of LOR and ENS were comparable. Lu $\boldsymbol{e t}$ al. ${ }^{(9)}$ reported that, psoas compartment block with the aid of US needed long time. In contrast, the present study findings were against the reported findings of other workers. Orebaugh et al. (10) after doing a retrospective analysis they reported that block performance times of interscalene, axillary, femoral and popliteal nerve blocks with the aid of combined ultrasound/electric nerve stimulation were shorter than the corresponding block performance times with the aid of nerve stimulation alone. Warman and Nicholls ${ }^{(11)}$ after conducting a level 2 evident study, the block performance time with the aid of US was shorter than that with the aid of nerve stimulation in many studies. Karmakar et al. (12) reported that the block performance time with the aid of combined ultrasound/electric nerve stimulation was shorter than that with the aid of nerve stimulation alone. The controversy between the of length of performance times with and without US aid in the present study findings and the other workers reported findings may be attributed to the different speeds of the operators (which depends on their experience level) in the adjustment of US machine and in imaging of the psoas muscle and the lumbar plexus and also to the different block depth which range from superficial or deep.

In the present study, it was found that patient discomfort scores during lumber plexus block with the aid of each of ENS and US/ENS were comparable and significantly higher than those with the aid of each of LOR and US and patient discomfort scores with the aid of each of LOR and US were comparable. Casati et al. 
(13) reported that patient discomfort score during multiple injection axillary brachial plexus block with the aid of nerve stimulation was significantly higher than that with the aid of US. Danelli et al. ${ }^{(14)}$ reported that, patient discomfort score during posterior lumber plexus block with the aid of nerve stimulation was significantly higher than that with the aid of ultrasound. Liu et al. ${ }^{(15)}$ after systematic review they reported that patient discomfort scores during the various regional blocks with the aid of US were significantly lower than those with the aid of other techniques. The higher patient discomfort scores in US/ENS than with the aid of LOR and US was attributed to that, electric nerve stimulation is painful and looks like paresthesia and this in agreement with Liguori et al. ${ }^{(16)}$ who reported that, the patient discomfort scores during interscalene brachial plexus blockade with the aid of electric nerve stimulation and paresthesia were comparable.

In the present study, the means of the numbers of the blocked nerves from both lumber and sacral plexuses at $30 \mathrm{~min}$ after injection of local anesthetic mixture examined by pin prick sensation in the dermatomes of each nerve distribution of the plexus in ENS, US and US/ENS groups were significantly higher than that in LOR group. The mean of the numbers of the blocked nerves in US and US/ENS groups were comparable and significantly higher than that in ENS group.

In the present study, the detected superiority of US/ENS and US over LOR and ENS in providing blocking large number of the lumber plexus nerves was attributed to the advantage of US in placing the tip of the block needle within the fascial plane in close to the lumber plexus roots and hence most of the injected local anesthetic volume become around them ${ }^{(17)}$. These results are consistent with Mannion et al. ${ }^{(18)}$ and Strid

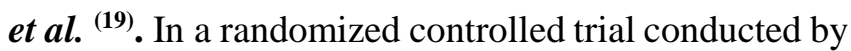
Bendtsen et al. (20), only one volunteer $(6 \%)$ in the SSPS group and none in the lumbar plexus block group $(0 \%)$ had complete sensory blockade of the dermatomes L2-S1, and this is attributed to difficulties like anatomical variations between individuals and ultrasound imaging optimization.

In the present study, success rates of PCB with the aid of ENS, US and US/ENS were significantly higher than that with the aid of LOR. Also, success rate with the aid of US and US/ENS were higher than that with the aid of each of ENS alone and success rate with the aid of US/ENS and significantly higher than that with the aid of US alone. These detected findings were in agreement with the reported finding of other workers (11, 21). Harato et al. ${ }^{(22)}$ reported that, posterior lumbar plexus block with the aid of US/ENS had a higher success rate than that with the aid of ENS alone. Also, Danelli et al. ${ }^{(8)}$ reported that, PCB success rate was higher in ENS group (80\%) than in LOR group (40\%).

In this study, the detected superiority dual US/ENS over US and the superiority of both over LOR and ENS in providing higher success rate during performing PCB was attributed to the combined advantages of US as it provides a real time delineation of the relevant anatomy and visualization of needle passage and distribution of the local anesthetic during the injection and the advantage of electric nerve stimulation as it provides a precise localization of nerve to be blocked.

In the present study, the onset of sensory and motor blocks in ENS, US and US/ENS groups were significantly shorter than that in LOR group. Also, the onset of sensory and motor blocks in US and US/ENS groups were comparable and shorter than that in ENS group. These present study findings were in agreement with the reported findings of some workers $(\mathbf{1 6}, \mathbf{2 3})$. Danelli et al. ${ }^{(8)}$ reported that, posterior lumber plexus block with the aid of nerve stimulation provided significant more rapid block onset than with the aid of loss of resistance $(12 \pm 6 \mathrm{~min}$ vs $22 \pm 6 \mathrm{~min}, \mathrm{P}=0.03)$.

In the present study, PCB produced strong intraoperative analgesia in the four groups (pain severity scores were comparable and this finding was in agreement with the finding of Stevens et al. ${ }^{(24)}$ who reported that posterior lumber plexus block provides a great reduction in pain scores and in the rescue" morphine boluses when used for postoperative pain relief. These findings were in agreement with the finding of Chan et al. (25), Dingemans et al. ${ }^{(26)}$ and Oberndorfer et $\boldsymbol{a l}$. ${ }^{(27)}$ In contrast, the present study findings were in controversy with other workers. Maalouf et al. (28) reported that, no significant difference in the need for postoperative supplemental systemic analgesia between the patients' group who received popliteal block with the aid of US and the patients' group who received popliteal block with the aid of ENS.

In the present study, it was found that, psoas compartment block with the aid of each of LOR, ENS, US and US/ENS was associated with stable hemodynamics. This finding was in agreement with the reported findings of Adali et al. ${ }^{(29)}$, Macel et al. ${ }^{\left({ }^{(3)}\right.}$ and Diwan et al. ${ }^{(31)}$ reported that the combined lumbar and sacral plexus block provides stable hemodynamics in the perioperative period and excellent postoperative analgesia after repair of hip fracture. The detected associated stability or minimal affection of patients' hemodynamic with PCB has been attributed to blocking somatic nerves without or with unilateral sympathectomy ${ }^{(32)}$.

In the present study, the detected complications of PCB were ipsilateral thoracic epidural block, contralateral femoral nerve block and sacral plexus block and vascular punctures in relatively few numbers of patients in each group. These findings were in partial agreement with Abrahams et al. ${ }^{(21)}$ and Harato et al. ${ }^{(22)}$. Sauter et al. ${ }^{(33)}$ reported that, posterior lumbar plexus block with the aid of combination of nerve stimulation and ultrasonography, allowed a precise localization of the plexus with less complications. 
In the present study, the absence of the occurrence of local anesthetic toxicity, spinal block and nerve injury was attributed to the precautions, which were taken during PCB performance as frequent negative aspiration (for blood and cerebrospinal fluid) during injection of local anesthetic, suspension of injection of local anesthetic against resistance and avoidance of local anesthetic injection if twitch response at $<0.3 \mathrm{~mA}$. This was in agreement with Amiri et al. finding ${ }^{(32)}$. They reported that, frequent negative aspiration during injection, suspension of injection against resistance and $<0.3 \mathrm{~mA}$ twitch response are three important key factors to avoid major complications in lumbar plexus block. A forceful injection during PCB may drive the LA through the vertebral foramina toward the epidural space. Contralateral spread anterior to the vertebral bodies has been described in thoracic paravertebral techniques and is another possibility. Claudio et al. ${ }^{(34)}$ reported that, in the absence of injection pressure monitoring, it is possible that injection pressures varied among previous research groups. This, in turn, may have contributed to the disparity in the published data regardless of the approach used, because subjective estimation of the force of injection is a poor predictor of actual injection force during injection of local anesthetic. In addition, a needle inserted or directed too medially may result in an injection into the epidural space itself ${ }^{(35)}$.

Limitations of the present study were the use of a relatively small sample size, not involving pediatric and geriatric patients in the study, the use of subjective injection pressure during injection of local anesthetic due to unavailability of pressure monitor, the use of a large volume $(40 \mathrm{ml})$ of local anesthetic, unavailability of ultrasound device in all hospitals and some anesthetists have no proficiency and experience in using ultrasound in regional anesthesia.

\section{CONCLUSION}

Usage of combined ultrasound and electric nerve stimulation was better than the individual usage of ultrasound, electric nerve stimulation, and loss of resistance in adequate localization of lumbar plexus nerves during psoas compartment block and the incidences of its associated complications were very low.

\section{RECOMMENDATION}

We recommend usage of combined ultrasound and electric nerve stimulation in peripheral nerve blocks to provide adequate nerve localization and visualization. Conflict of interest: No conflict of interest between authors.

\section{REFERENCES}

1. Prasad G, Khanna S, Jaishree S (2020): Review of adjuvants to local anesthetics in peripheral nerve blocks: Current and future trends. Saudi Journal of Anesthesia, 14(1):77-83.
2. Capdevila X, Biboulet P, Morau D et al. (2008): How and why to use ultrasound for regional blockade. Acta Anaesthesiol Belg., 59(3):147-54.

3. Pandin P, Vandesteene A, d'Hollander A (2002): Lumbar plexus posterior approach: a catheter placement description using electrical nerve stimulation. Anesthesia \& Analgesia, 95(5):1428-31.

4. Ilfeld B, Loland V, Mariano E (2010): Prepuncture ultrasound imaging to predict transverse process and lumbar plexus depth for psoas compartment block and perineural catheter insertion: a prospective, observational study. Anesthesia \& Analgesia., 110(6):1725-32.

5. Kirchmair L, Entner T, Wissel J et al. (2001): A study of the paravertebral anatomy for ultrasound-guided posterior lumbar plexus block. Anesthesia \& Analgesia., 93(2):477-81.

6. Zhang X, Li Y, He W et al. (2019): Combined ultrasound and nerve stimulator-guided deep nerve block may decrease the rate of local anesthetics systemic toxicity: a randomized clinical trial. BMC Anesthesiology, 19(1):103-105.

7. Kumar L, Sharma C, Sibi M et al. (2014): Comparison of peripheral nerve stimulator versus ultrasonography guided axillary block using multiple injection technique. Indian Journal of Anesthesia, 58(6):700-6.

8. Danelli G, Ghisi D, Bellinghieri F et al. (2011): The nerve stimulation technique versus the loss of resistance technique for the posterior approach to lumbar plexus block: a randomized, prospective, observer-blinded, pilot study. Minerva Anestesiologica, 77(10):959-63.

9. Lu R, Shen C, Yang C et al. (2018): Comparison of lumbar plexus block using the short axis in-plane method at the plane of the transverse process and at the articular process: a randomized controlled trial. BMC Anesthesiology, 18(1):17-23.

10. Orebaugh S, Williams B, Kentor M (2007): Ultrasound guidance with nerve stimulation reduces the time necessary for resident peripheral nerve blockade. Regional Anesthesia \& Pain Medicine, 32(5):448-54.

11. Warman P, Nicholls B (2009): Ultrasound-guided nerve blocks: efficacy and safety. Best Practice \& Research Clinical Anaesthesiology, 23(3):313-26.

12. Karmakar M, Li J, Kwok W et al. (2015): Ultrasoundguided lumbar plexus block using a transverse scan through the lumbar intertransverse space: a prospective case series. Regional Anesthesia \& Pain Medicine, 40(1):75-81.

13. Casati A, Danelli G, Baciarello M et al. (2007): A prospective, randomized comparison between ultrasound and nerve stimulation guidance for multiple injection axillary brachial plexus block. Anesthesiology: The Journal of the American Society of Anesthesiologists, 106(5):992-6.

14. Danelli G, Fanelli A, Ghisi D et al. (2009): Ultrasound vs nerve stimulation multiple injection technique for posterior popliteal sciatic nerve block. Anesthesia, 64(6):638-42.

15. Liu S, Ngeow J, Yadeau J (2009): Ultrasound-guided regional anesthesia and analgesia: a qualitative systematic review'. Regional Anesthesia and Pain Medicine, 34(1): 47-59.

16. Liguori G, Zayas V, YaDeau J et al. (2006): Nerve localization techniques for interscalene brachial plexus blockade: a prospective, randomized comparison of 
mechanical paresthesia versus electrical stimulation. Anesthesia \& Analgesia, 103(3):761-7.

17. Marhofer P, Greher M, Kapral S (2005): Ultrasound guidance in regional anesthesia. British Journal of Anesthesia, 94(1):7-17.

18. Mannion S, Barrett J, Kelly D et al. (2005): A description of the spread of injectate after psoas compartment block using magnetic resonance imaging. Regional Anesthesia \& Pain Medicine, 30(6):567-71.

19. Strid J, Sauter A, Ullensvang $K$ et al. (2017): Ultrasound-guided lumbar plexus block in volunteers; a randomized controlled trial. BJA: British Journal of Anesthesia, 118(3):430-8.

20. Bendtsen T, Pedersen E, Haroutounian S et al. (2014): The suprasacral parallel shift vs lumbar plexus blockade with ultrasound guidance in healthy volunteers-a randomised controlled trial. Anesthesia, 69(11):1227-40.

21. Abrahams M, Aziz M, Fu R et al. (2009): Ultrasound guidance compared with electrical neurostimulation for peripheral nerve block: a systematic review and metaanalysis of randomized controlled trials. British Journal of Anesthesia, 102(3):408-17.

22. Harato M, Hiroshi I, Manabu A et al. (2010): Current status and future of ultrasound-guided nerve block for the lumbar region and sacral region - Nerve stimulation alone and ultrasonic guide for lumbar plexus block and parasacral block - comparison of combined nerve stimulation methods. Journal of the Japanese Society of Clinical Anesthesia, 30(7): 952-8.

23. Antonakakis J, Ting P, Sites B (2011): Ultrasoundguided regional anesthesia for peripheral nerve blocks: an evidence-based outcome review. Anesthesiology Clinics, 29(2):179-91.

24. Stevens R, Van Gessel E, Flory $\mathbf{N}$ et al. (2000): Lumbar plexus block reduces pain and blood loss associated with total hip arthroplasty. Anesthesiology: The Journal of the American Society of Anesthesiologists, 93(1):115-21.

25. Chan V, Perlas A, McCartney C et al. (2007): Ultrasound guidance improves success rate of axillary brachial plexus block. Canadian Journal of Anesthesia, 54(3):176-82.
26. Dingemans E, Williams S, Arcand G et al. (2007): Neurostimulation in ultrasound-guided infraclavicular block: a prospective randomized trial. Anesthesia \& Analgesia, 104(5):1275-80.

27. Oberndorfer U, Marhofer P, Bösenberg A et al. (2007): Ultrasonographic guidance for sciatic and femoral nerve blocks in children. British Journal of Anesthesia, 98(6):797-801.

28. Maalouf D, Liu S, Movahedi $\mathrm{R}$ et al. (2012): Nerve stimulator versus ultrasound guidance for placement of popliteal catheters for foot and ankle surgery. Journal of Clinical Anesthesia, 24(1):44-50.

29. Adali S, Erkalp K, Erden V et al. (2011): Spinal anesthesia and combined sciatic nerve/lumbar plexus block techniques in lower extremity orthopedic surgery. Acta Orthop Traumatol Turc., 45(4):225-32.

30. Macel D, Slagt C, Hoeksema M et al. (2011): Hemodynamic changes during a combined psoas compartment-sciatic nerve block for elective orthopedic surgery. Anesthesia \& Analgesia, 112(3):719-24.

31. Diwan S, Pradhan C, Patil A et al. (2009): Combined lumbar and sacral plexus block in geriatric high-risk patients undergoing an awake repair of fracture intertrochanteric of femur anesthesia', Journal of Anesthesia and Critical Care Case Reports, 4(1): 21-30.

32. Amiri H, Zamani M, Safari S (2014): Lumbar plexus block for management of hip surgeries. Anesthesiology and Pain Medicine, 4(3): 19407.

33. Sauter A, Ullensvang K, Niemi G et al. (2015): The Shamrock lumbar plexus block: a dose-finding study. European Journal of Anaesthesiology (EJA), 32(11):76470.

34. Claudio R, Hadzic A, Shih $H$ et al. (2004): Injection pressures by anesthesiologists during simulated peripheral nerve block. Regional Anesthesia \& Pain Medicine, 29(3):201-5.

35. Mannion S, O'Callaghan S, Walsh M et al. (2005): In with the new, out with the old? Comparison of two approaches for psoas compartment block. Anesthesia \& Analgesia, 101(1):259-64. 